Tôhoku Math. Journ. 35 (1983), 597-605.

\title{
STABILITY PROPERTIES FOR FUNCTIONAL DIFFERENTIAL EQUATIONS WITH INFINITE DELAY
}

\author{
Dedicated to Professor Tamotsu Tsuchikura on his sixtieth birthday
}

\section{YoSHIYUKI Hino}

(Received December 9, 1982, revised February 21, 1983)

1. Introduction. For ordinary differential equations, there are many results on stability properties and limiting equations by using the techniques of topological dynamics (cf. [8], [10], [11]). Furthermore, Bondi, Moauro and Visentin [2] and Artstein [1] gave some of the results as above by assuming the uniqueness of solutions of limiting equations for the initial conditions and by using standard arguments based on the fact that the solution operator is completely continuous.

In this paper, more generally, we shall discuss functional differential equations with infinite delay, for which it is known that the solution operator is not necessarily completely continuous but an $\alpha$-contraction (cf. [3], [4]). We extend some of the results mentioned above for ordinary differential equations; that is, we show that some stability properties of a bounded solution of a given equation follow from stability properties of its limiting equations without the uniqueness of the solution of limiting equations for the initial conditions. In particular, our results contain the following: for a periodic system, if a bounded solution is uniformly asymptotically stable, then it is totally stable.

2. Phase space $B$, notations and definitions. First, we shall give the space $B$ discussed by Kato [7] (see also [3], [4]). Let $|x|$ be any norm of $x$ in $R^{n}$. Let $B$ be a real linear vector space of functions mapping $(-\infty, 0]$ into $R^{n}$ with a semi-norm $|\cdot|_{B}$. If $x$ is a function defined on $(-\infty, a)$, then for each $t \in(-\infty, a)$ we define the function $x_{t}$ by the relation $x_{t}(s)=x(t+s),-\infty<s \leqq 0$. The space $\mathrm{B}$ is assumed to have the following properties:

( I ) If $x(t)$ is defined on $(-\infty, a)$, continuous on $[\sigma, a), \sigma<a$, and $x_{\sigma} \in B$, then for $t \in[\sigma, a)$ we have:

$$
\text { (I. 1) } x_{t} \in B \text {. }
$$

This research was partially supported by Grant-in-Aid for Scientific Research (No, 57540058), Ministry of Education. 
(I. 2) $x_{t}$ is continuous in $t$ with respect to $|\cdot|_{B}$.

(I. 3) there are a $K>0$ and a positive continuous function $M(\beta)$, $M(\beta) \rightarrow 0$ as $\beta \rightarrow \infty$, such that $\left|x_{t}\right|_{B} \leqq K \sup _{\sigma \leqq \theta \leqq t}|x(\theta)|+M(t-\sigma)\left|x_{\sigma}\right|_{B}$.

(II) $|\phi(0)| \leqq M_{1}|\phi|_{B}$ for a constant $M_{1}>0$.

REMARK 1. Hale and Kato [4] have given hypotheses on the space $B$ in a slightly different way. However, in our present context, there is no difference between the two.

Let $S$ be a compact subset in $B$ and let $\alpha>0$ and $\beta>0$. Denote by $X(S, \alpha, \beta)$ the set $\left\{x_{t} \mid t \geqq 0\right.$ and $x(\cdot)$ is such that $x_{0} \in S,|x(\theta)| \leqq \alpha$ for $\theta \in[0, \infty)$ and $\left|x\left(\theta^{1}\right)-x\left(\theta^{2}\right)\right| \leqq \beta\left|\theta^{1}-\theta^{2}\right|$ for $\left.\theta^{1}, \theta^{2} \in[0, \infty)\right\}$.

Lemma 1. ([4, Corollary 3.2]). The closure $X(S, \alpha, \beta)^{-}$of $X(S, \alpha, \beta)$ is compact in $B$.

Throughout this paper we shall suppose that $F(t, \phi)$ is an $R^{n}$-valued function on $R \times B, R=(-\infty, \infty)$, and for any compact set $S \subset B, F(t, \phi)$ is bounded and uniformly continuous on $R \times S$. Then, it is known that for any compact set $W \subset R \times B$, any sequence $\left\{t_{n}^{\prime}\right\}, t_{n}^{\prime} \geqq 0$, contains a subsequence $\left\{t_{n}\right\}$ such that $\left\{F\left(t+t_{n}, \phi\right)\right\}$ converges uniformly for $(t, \phi) \in$ $W$ (cf. [4], [6]). The hull $H(F)$ (resp. $H^{+}(F)$ ) denotes the set of pairs $(G, \Omega), \Omega \subset R \times B$, such that there exists a sequence $\left\{t_{n}\right\}, t_{n} \geqq 0$ (resp. $t_{n} \rightarrow$ $\infty$ as $n \rightarrow \infty)$, such that $\left\{F\left(t+t_{n}, \phi\right)\right\}$ converges to $G(t, \phi)$ for $(t, \phi) \in \Omega$.

REMARK 2. We shall note that if $(G, \Omega) \in H(F)$, then for any compact set $S \subset B$, there exists a $\left(G^{*}, \Omega^{*}\right) \in H(F)$ such that $\Omega^{*} \supset \Omega \cup\{I \times S\}$ and $G^{*}(t, \phi)=G(t, \phi)$ on $\Omega$, where $I=[0, \infty)$, because $I \times S$ is separable (see [4], [6]).

Consider the system of functional differential equations

$$
\dot{x}(t)=F\left(t, x_{t}\right),
$$

where $\dot{x}(t)$ denotes the right hand derivative. Let $x(t, F)$ be a solution of (1). In particular, let $x\left(t, s, \phi^{0}, F\right)$ be a solution of (1) through $\left(s, \phi^{0}\right)$. We assume that System (1) has a bounded solution $u(t)$ defined on $I$ and that $L=\sup \left\{\left.|F(t, \phi)||t \geqq 0,| \phi\right|_{B} \leqq 2 H\right\}<\infty$, where $H$ is a positive constant which satisfies $\left|u_{t}\right|_{B} \leqq H$ for $t>0$. Therefore by noting that $\left\{u_{t} \mid t \geqq 0\right\}^{-}$is contained in the compact set $X\left(\left\{u_{0}\right\}, M_{1} H, L\right)^{-}$(see Lemma $1)$, we may assume that for any $(G, \Omega) \in H(F)$, we have $\Omega \supset I \times\left\{u_{t} \mid t \geqq 0\right\}^{-}$ by Remark 2 . Hence it is easily shown that for any $(v, G, \Omega) \in H(u, F)$ the function $v(t)$ is a solution of

$$
\dot{x}(t)=G\left(t, x_{t}\right)
$$


defined on $I$ (cf. [4]). Here and henceforth, the hulls $H(u)\left(\right.$ resp. $\left.H^{+}(u)\right)$ and $H(u, F)$ (resp. $H^{+}(u, F)$ ) are defined in the same way as $H(F)$ (resp. $H^{+}(F)$ ). We say that System (1) is regular if, for any $(G, \Omega) \in H(F)$, every solution of (2) is unique for the initial conditions.

We shall give several definitions of stabilities.

Definition 1. The solution $u(t)$ is uniformly stable, if for any $\varepsilon>$ 0 , there exists a $\delta(\varepsilon)>0$ such that $\left|x_{t}\left(s, \phi^{0}, F\right)-u_{t}\right|_{B}<\varepsilon$ for all $t \geqq s$, whenever $\left|\phi^{0}-u_{s}\right|_{B}<\delta(\varepsilon)$ for some $s \geqq 0$.

DeFinition 2. The solution $u(t)$ is weakly uniformly asymptotically stable, if it is uniformly stable and if there exists a $\delta_{0}>0$ such that $\left|x_{t}\left(s, \phi^{0}, F\right)-u_{t}\right|_{B} \rightarrow 0$ as $t \rightarrow \infty$, whenever $\left|\phi^{0}-u_{s}\right|_{B}<\delta_{0}$ for some $s \geqq 0$.

Definition 3. The solution $u(t)$ is uniformly asymptotically stable, if it is uniformly stable and if there exists a $\delta_{0}>0$ and for any $\varepsilon>0$ there exists a $T(\varepsilon)>0$ such that $\left|x_{t}\left(s, \phi^{0}, F\right)-u_{t}\right|_{B}<\varepsilon$ for $t \geqq s+T(\varepsilon)$, whenever $\left|\phi^{0}-u_{s}\right|_{B}<\delta_{0}$ for some $s \geqq 0$.

Definition 4. The solution $u(t)$ is uniformly stable in $H(F)$ (resp. $H^{+}(F)$ ), if for any $\varepsilon>0$, there exists a $\delta(\varepsilon)>0$ such that for any $s \in I$ and $(v, G, \Omega) \in H(u, F)\left(\right.$ resp. $\left.H^{+}(u, F)\right),\left|\phi^{0}-v_{s}\right|_{B}<\delta(\varepsilon)$ implies $\mid x_{t}\left(s, \phi^{0}, G\right)-$ $\left.v_{t}\right|_{B}<\varepsilon$ for all $t \geqq s$.

Definition 5. The solution $u(t)$ is attracting in $H(F)$ (resp. $H^{+}(F)$ ), if there exists a $\delta_{0}>0$ such that for any $s \in I$ and $(v, G, \Omega) \in H(u, F)$ (resp. $\left.H^{+}(u, F)\right),\left|\phi^{0}-v_{s}\right|_{B}<\delta_{0}$ implies $\left|x_{t}\left(s, \phi^{0}, G\right)-v_{t}\right|_{B} \rightarrow 0$ as $t \rightarrow \infty$.

DeFinition 6. The solution $u(t)$ is weakly uniformly asymptotically stable in $H(F)$ (resp. $H^{+}(F)$ ), if it is uniformly stable in $H(F)$ (resp. $\left.H^{+}(F)\right)$ and attracting in $H(F)$ (resp. $H^{+}(F)$ ).

DeFinition 7. The solution $u(t)$ is uniformly attracting in $H(F)$ (resp. $H^{+}(F)$ ), if there exists a $\delta_{0}>0$ and for any $\varepsilon>0$ there exists a $T(\varepsilon)>0$ such that for any $s \in I$ and $(v, G, \Omega) \in H(u, F)$ (resp. $H^{+}(u, F)$ ), $\left|\phi^{0}-v_{s}\right|_{B}<\delta_{0}$ implies $\left|x_{t}\left(s, \phi^{0}, G\right)-v_{t}\right|_{B}<\varepsilon$ for $t \geqq s+T(\varepsilon)$.

Definition 8. The solution $u(t)$ is uniformly asymptotically stable in $H(F)$ (resp. $H^{+}(F)$ ), if it is uniformly stable in $H(F)$ (resp. $H^{+}(F)$ ) and uniformly attracting in $H(F)$ (resp. $H^{+}(F)$ ).

Definition 9. The solution $u(t)$ is totally stable, if for any $\varepsilon>0$ there exists a $\delta(\varepsilon)>0$ such that if $g(t)$ is continuous and satisfies $|g(t)|<$ $\delta(\varepsilon)$ on $[0, \infty)$ and if $\left|\phi^{0}-u_{s}\right|_{B}<\delta(\varepsilon)$ for an $s \in I$, then $\mid x_{t}\left(s, \phi^{0}, F+g\right)-$ $\left.u_{t}\right|_{B}<\varepsilon$ for all $t \geqq s$, where $x\left(t, s, \phi^{0}, F+g\right)$ is a solution of 


$$
\dot{x}(t)=F\left(t, x_{t}\right)+g(t)
$$

through $\left(s, \dot{\phi}^{0}\right)$.

REMARK 3. In the above concepts, if the semi-norm $\left|x_{t}-u_{t}\right|_{B}$ (or $\left.\left|x_{t}-v_{t}\right|_{B}\right)$ is replaced by the $R^{n}$-norm, then the concepts of the stabilities in $R^{n}$ will be obtained. However, it is known that the concepts of stabilities given in the above and stabilities in $R^{n}$ are equivalent under the hypotheses (I) and (II) (see [7, Theorem 5]).

The following Propositions 1 and 2 are well known for ordinary differential equations and can be proved by using the parallel arguments as in the proof of Theorem 13.3 and Theorem 13.2 in [12], respectively. These show that some stability properties of limiting equations follow from stability properties of (1) under suitable conditions.

Proposition 1. Assume that System (1) is regular. If the solution $u(t)$ is uniformly stable (resp. uniformly asymptotically stable), then it is uniformly stable in $H(F)$ (resp. uniformly asymptotically stable in $H(F))$.

Proposition 2. If $F(t, \phi)$ is periodic in $t$, then the solution $u(t)$ is uniformly stable in $H(F)$ (resp. weakly uniformly asymptotically stable in $H(F)$, resp. uniformly asymptotically stable in $H(F)$ ), if it is uniformly stable (resp. weakly uniformly asymptotically stable, resp. uniformly asymptotically stable).

An inheritance of weak uniform asymptotic stability for almost periodic systems will be discussed in Section 5 .

3. Main theorem and related results. We shall give our theorem, whose proof will be given in Section 4.

THEOREM. If the solution $u(t)$ is unique for the initial conditions and weakly uniformly asymptotically stable in $H^{+}(F)$, then it is uniformly asymptotically stable in $H(F)$ and totally stable.

REMARK 4. For ordinary differential equations, the "if" part of Theorem $\mathrm{F}$ in [1] is related to our theorem, in the case where $u(t)=0$. However, there are some differences between the two, because the concept of limiting equations defined by Artstein covers a wide family of functions compared with Sell's concept (our concept is a natural extension of Sell's to functional differential equations with infinite delay) and Artstein has assumed that any solution of every limiting equation is unique for the initial conditions and that the null solution of a given equation is unique for the initial conditions and uniformly attracting 
with respect to every limiting equation.

By Propositions 1 and 2 and Theorem, we have the following corollaries. For ordinary differential equations, Corollaries 1,2 and 3 are proved by using the standard arguments (cf. [12]), because the solution operator is completely continuous.

CoRollary 1. Assume that System (1) is regular. If $u(t)$ is uniformly asymptotically stable, then it is totally stable.

Corollary 2. Assume that $F(t, \phi)$ is periodic in $t$. Then $u(t)$ is weakly uniformly asymptotically stable if and only if it is uniformly asymptotically stable.

CoROLlary 3. Assume that $F(t, \phi)$ is periodic in $t$. If $u(t)$ is weakly uniformly asymptotically stable, then it is totally stable.

REMARK 5. For ordinary differential equations, Bondi, Moauro and Visentin [2, Theorem 3.1] have shown that if $F(t, x)$ satisfies a Lipschitz condition and the null solution of $\dot{x}(t)=F(t, x)$ is uniformly attracting in $H^{+}(F)$, then the null solution is uniformly asymptotically stable in $H(F)$. However, to such an equation whose limiting equation is $\dot{x}(t)=$ $-x^{1 / 3},[2$, Theorem 3.1] is not applicable but our theorem is.

We shall consider the perturbed system, that is,

$$
\dot{x}(t)=F\left(t, x_{t}\right)+a\left(t, x_{t}\right),
$$

where $a(t, \phi)$ is continuous on $I \times B$ and for any compact set $S \subset B$, $a(t, \phi) \rightarrow 0$ as $t \rightarrow \infty$ uniformly on $S$.

COROLLARY 4. Suppose that Equation (4) has the null solution which is unique for the initial conditions and that System (1) is regular. Then the null solution of (4) is uniformly asymptotically stable and totally stable, if the null solution of (1) is uniformly asymptotically stable.

Ppoof. The null solution of (1) is uniformly asymptotically stable, $H^{+}(F)=H^{+}(F+a)$ and System (1) is regular. Hence for any $(G, \Omega) \in$ $H^{+}(F+a)$, the null solution of (2) is uniformly asymptotically stable with the common $\left(\delta(\cdot), \delta_{0} . T(\cdot)\right)$ as the one for the null solution of (1) by Proposition 1. Hence we have the conclusion by Theorem, because the null solution of (4) is unique for the initial conditions.

4. Proof of Theorem. We shall use the following lemma to prove Theorem, which corresponds to [5, Lemma 2] and [6, Lemma 1] for functional differential equations. 
LEMma 2. Assume that any solution in $H(u)$ is unique for the initial conditions. Let $T>0$. Then for any $\varepsilon>0$, there exists $a \delta(\varepsilon)>$ 0 such that for any $s \in I$, if $\left|\phi^{0}-u_{s}\right|_{B}<\delta(\varepsilon)$ and $|g(t)|<\delta(\varepsilon)$ on $[s, s+T]$, then $\left|x_{t}\left(s, \phi^{0}, F+g\right)-u_{t}\right|_{B}<\varepsilon$ for $t \in[s, s+T]$.

Proof. Suppose the contrary. Then, for some $\varepsilon, H>\varepsilon>0$, there exist sequences $\left\{t_{n}\right\}, t_{n} \in I,\left\{g^{n}(t)\right\}$ and $\left\{\tau_{n}\right\}$ such that $\left|g^{n}(t)\right|<1 / n$ for $t \in$ $\left[t_{n}, t_{n}+T\right],\left|x_{t_{n}}^{n}\left(F+g^{n}\right)-u_{t_{n}}\right|_{B}<1 / n, \tau_{n} \in\left[t_{n}, t_{n}+T\right],\left|x_{t}^{n}\left(F+g^{n}\right)-u_{t}\right|_{B}<$ $\varepsilon$ for $t \in\left[t_{n}, \tau_{n}\right)$ and $\left|x_{\tau_{n}}^{n}(F+g)-u_{\tau_{n}}\right|_{B}=\varepsilon$. Since $\tau_{n}-t_{n} \in[0, T]$, we can assume that $p_{n}=\tau_{n}-t_{n}$ converges to $p \in[0, T]$ as $n \rightarrow \infty$. Set

$$
y^{n}(t)= \begin{cases}x^{n}\left(t+t_{n}, F+g^{n}\right), & t \in\left(-\infty, p_{n}\right), \\ x^{n}\left(\tau_{n}, F+g^{n}\right), & t \in\left[p_{n}, T\right] .\end{cases}
$$

Then $y^{n}(t)$ is the solution of $\dot{x}(t)=F\left(t+t_{n}, x_{t}\right)+g^{n}\left(t+t_{n}\right)$ on $\left[0, p_{n}\right]$ such that $y_{0}^{n}=x_{t_{n}}^{n}\left(F+g^{n}\right)$. Since $\left|y_{t}^{n}\right|_{B} \leqq\left|y_{t}^{n}-u_{t}\right|_{B}+\left|u_{t}\right|_{B} \leqq \mid x_{t+t_{n}}^{n}\left(F+g^{n}\right)-$ $\left.u_{t}\right|_{B}+\left|u_{t}\right|_{B}<\varepsilon+H<2 H$ for $t \in\left[0, p_{n}\right]$ and $\left|\dot{y}^{n}(t)\right| \leqq\left|F\left(t+t_{n}, y_{t}^{n}\right)\right|+$ $\left|g^{n}\left(t+t_{n}\right)\right|<L+1<2 L$ for $t \in\left[0, p_{n}\right)$ and $\left|\dot{y}^{n}(t)\right|=0$ for $t \in\left[p_{n}, T\right]$, we may assume that $y_{t}^{n} \in X\left(S, 2 M_{1} H, 2 L\right)^{-}$, where $S=\left\{x_{t_{n}}^{n}\left(F+g^{n}\right) \mid n=1,2,3, \cdots\right\}^{-}$ is compact in $B$. Hence we can assume that $F\left(t+t_{n}, \phi\right)+g^{n}\left(t+t_{n}\right) \rightarrow$ $G(t, \phi)$ as $n \rightarrow \infty$ uniformly on $[0, p] \times X\left(S \cup\left\{u_{0}\right\}, 2 M_{1} H, 2 L\right)^{-}$and $y^{n}(t)$ and $u\left(t+t_{n}\right)$ converge to solutions $y(t)$ and $v(t)$ of (2) as $n \rightarrow \infty$ uniformly on $[0, p]$, respectively. $y_{0}=v_{0}$ implies $\left|y_{t}-v_{t}\right|_{B}=0$ on $[0, p]$, because $v(t)$ is unique for the initial conditions. However $\left|y_{p_{n}}^{n}-u_{p_{n}+t_{n}}\right|_{B}=$ $\left|x_{p_{n}+t_{n}}^{n}\left(F+g^{n}\right)-u_{p_{n}+t_{n}}\right|_{B}=\left|x_{\tau_{n}}^{n}\left(F+g^{n}\right)-u_{\tau_{n}}\right|_{B}=\varepsilon$ implies $\left|y_{p}-v_{p}\right|_{B}=\varepsilon$, which is a contradiction.

Proof of THEOREM. It is known that if $u(t)$ is uniformly stable and attracting in $H^{+}(F)$, then it is uniformly asymptotically stable ([4, Theorem 6.2]). Hence we shall show only that the solution $u(t)$ is totally stable, because the total stability implies the uniform stability. Suppose not. Then there are sequences $\left\{t_{n}\right\}, t_{n} \geqq 0,\left\{r_{n}\right\}, r_{n}>0,\left\{g^{n}(t)\right\}$ and $\left\{x^{n}(t\right.$, $\left.\left.F+g^{n}\right)\right\}$ and a constant $\delta_{1}, \delta_{1}<\min \left\{H, \delta_{0} / 2\right\}$, such that

$$
\left|x_{t_{n}}^{n}\left(F+g^{n}\right)-u_{t_{n}}\right|_{B}<1 / n \text { and }\left|g^{n}(t)\right|<1 / n \text { on }\left[t_{n}, \infty\right)
$$

and

$$
\begin{aligned}
& \left|x_{t_{n}+r_{n}}^{n}\left(F+g^{n}\right)-u_{t_{n}+r_{n}}\right|_{B}=\delta_{1} \text { and }\left|x_{t}^{n}\left(F+g^{n}\right)-u_{t}\right|_{B}<\delta_{1} \\
& \text { on . }\left[t_{n}, t_{n}+r_{n}\right)
\end{aligned}
$$

where $\delta_{0}$ is the one given for the attraction in $H^{+}(F)$ of $u(t)$. There exists a sequence $\left\{q_{n}\right\}, 0<q_{n}<r_{n}$, such that

$$
\left|x_{t_{n}+q_{n}}^{n}\left(F+g^{n}\right)-u_{t_{n}+q_{n}}\right|_{B}=\delta\left(\delta_{1} / 2\right) / 2
$$


and

$$
\delta\left(\delta_{1} / 2\right) / 2 \leqq\left|x_{t}^{n}\left(F+g^{n}\right)-u_{t}\right|_{B} \leqq \delta_{1} \quad \text { on }\left[t_{n}+q_{n}, t_{n}+r_{n}\right]
$$

for a large $n$ by (5) and (6), where $\delta(\cdot)$ is the one given for uniform stability in $H^{+}(F)$ of $u(t)$. Suppose that there exists a subsequence of $\left\{q_{n}\right\}$, which we shall denote by $\left\{q_{n}\right\}$ again, such that $q_{n}$ converges to some $q, q \in I$. Since any solution in $H(u)$ is uniqe for initial conditions, it follows from (5) that there exists an $n_{0}>0$ such that for any $n \geqq$ $n_{0}, q+1 \geqq q_{n} \geqq 0$ and $\left|x_{t_{n}+t}^{n}\left(F+g^{n}\right)-u_{t_{n}+t}\right|_{B}<\delta\left(\delta_{1} / 4\right) / 4$ for $t \in[0, q+1]$ by Lemma 2 , which contradicts (7). Therefore, we can see that $q_{n} \rightarrow \infty$ as $n \rightarrow \infty$.

Put $p_{n}=r_{n}-q_{n}$ and suppose that $p_{n} \rightarrow \infty$ as $n \rightarrow \infty$. Set $s_{n}=q_{n}+$ $\left(p_{n} / 2\right)$ and $z^{n}(t)=x^{n}\left(t+t_{n}+s_{n}, F+g^{n}\right)$. Then, we have $z_{t}^{n} \in X\left(S, 2 M_{1} H\right.$, $2 L)^{-}$for $t \in\left[-p_{n} / 2, p_{n} / 2\right]$, where $S=\left\{x_{t_{n}+q_{n}}\left(F+g^{n}\right) \mid n=1,2,3, \cdots\right\}^{-}$, because $z_{-p_{n} / 2}^{n}=x_{t_{n}+q_{n}}^{n}\left(F+g^{n}\right) \in S$ and $\left|z_{t}^{n}\right|_{B}=\left|x_{t+t_{n}+s_{n}}^{n}\left(F+g^{n}\right)\right|_{B}=$ $\left|x_{t+t_{n}+s_{n}}^{n}\left(F+g^{n}\right)-u_{t+t_{n}+s_{n}}\right|_{B}+\left|u_{t+t_{n}+s_{n}}\right|_{B}<\delta_{1}+H<2 H$ for $t \in\left[-p_{n} / 2\right.$, $\left.p_{n} / 2\right]$ by (8). Clearly, $z^{n}(t)$ and $u\left(t+t_{n}+s_{n}\right)$ are solutions of $\dot{x}(t)=$ $F\left(t+t_{n}+s_{n}, x_{t}\right)+g^{n}\left(t+t_{n}+s_{n}\right)$ and $\dot{x}(t)=F\left(t+t_{n}+s_{n}, x_{t}\right)$, respectively. Hence we can easily show that there are a function. $P(t, \phi)$ and solutions $z(t)$ and $v(t)$ of $\dot{x}(t)=P\left(t, x_{t}\right)$ defined on $I$ such that $F\left(t+t_{n}+s_{n}, \phi\right)+$ $g^{n}\left(t+t_{n}+s_{n}\right) \rightarrow P(t, \phi)$ uniformly on (any compact set of $\left.I\right) \times X(S \cup$ $\left.\left\{u_{0}\right\}, 2 M_{1} H, 2 L\right)^{-}$as $n \rightarrow \infty$ and $z^{n}(t) \rightarrow z(t)$ and $u\left(t+t_{n}+s_{n}\right) \rightarrow v(t)$ uniformly on any compact subset of $I$ as $n \rightarrow \infty$, taking a subsequence, if necessary. For a fixed $t>0$, there exists an $n_{1}>0$ such that for every $n \geqq n_{1}, r_{n}-s_{n}=p_{n} / 2>t$, because $p_{n} \rightarrow \infty$ as $n \rightarrow \infty$. Hence for $n \geqq n_{1}$, we have $q_{n}<t+s_{n}<r_{n}$. Thus for $n \geqq n_{1}$,

$$
\left|z_{t}^{n}-u_{t+t_{n}+s_{n}}\right|_{B} \geqq \delta\left(\delta_{1} / 2\right) / 2
$$

by (8). There exists an $n_{2}>n_{1}$ such that for every $n \geqq n_{2}$

$$
\left|z_{t}^{n}-z_{t}\right|_{B} \leqq \delta\left(\delta_{1} / 2\right) / 8 \text { and }\left|u_{t+t_{n}+s_{n}}-v_{t}\right|_{B} \leqq \delta\left(\delta_{1} / 2\right) / 8 \text {. }
$$

By (9) and (10), for every $n \geqq n_{2}$

$$
\begin{aligned}
\left|z_{t}-v_{t}\right|_{B} & \geqq\left|z_{t}^{n}-u_{t+t_{n}+s_{n}}\right|_{B}-\left|u_{t+t_{n}+s_{n}}-v_{t}\right|_{B}-\left|z_{t}^{n}-z_{t}\right|_{B} \\
& \geqq \delta\left(\delta_{1} / 2\right) / 4 .
\end{aligned}
$$

However, $\left|z_{0}-v_{0}\right|_{B}<\delta_{0}$ implies $\left|z_{t}-v_{t}\right|_{B} \rightarrow 0$ as $t \rightarrow \infty$, which is a contradiction of (11). We can show that $\left\{p_{n}\right\}$ is bounded.

Hence we may assume that $p_{n}$ converges to $p, p \in I$, as $n \rightarrow \infty$ and $0 \leqq p_{n}<p+1$ for all $n$. Set

$$
y^{n}(t)= \begin{cases}x^{n}\left(t+t_{n}+q_{n}, F+g^{n}\right), & t \in\left(-\infty, p_{n}\right), \\ x^{n}\left(t_{n}+r_{n}, F+g^{n}\right), & t \in\left[p_{n}, p+1\right] .\end{cases}
$$


Then, by using the same arguments as in the proof of Lemma 2, we may assume that $F\left(t+t_{n}+q_{n}, \phi\right)+g^{n}\left(t+t_{n}+q_{n}\right) \rightarrow G(t, \phi)$ uniformly on $[0, p] \times X\left(S \cup\left\{u_{0}\right\}, 2 M_{1} H, 2 L\right)^{-}$as $n \rightarrow \infty$ and that $y^{n}(t)$ and $u\left(t+t_{n}+\right.$ $q_{n}$ ) converge to solutions $y(t)$ and $w(t)$ of (2) as $n \rightarrow \infty$ uniformly on $[0, p]$, respectively. Since $\left|y_{0}-w_{0}\right|_{B}=\delta\left(\delta_{1} / 2\right) / 2$ by $(7)$, we have $\mid y_{p}-$ $\left.w_{p}\right|_{B}<\delta_{1} / 2$. However, we have a contradiction by (6), because $\mid y_{p_{n}}^{n}-$ $\left.y_{p}^{n}\right|_{B}=\left|x_{p_{n}+t_{n}+q_{n}}^{n}\left(F+g^{n}\right)-x_{p+t_{n}+q_{n}}^{n}\left(F+g^{n}\right)\right|_{B} \leqq K \sup _{-\left(p_{n}+q_{n}\right) \leqq \theta \leq 0} \mid x^{n}\left(p_{n}+\right.$ $\left.t_{n}+q_{n}+\theta, F+g^{n}\right)-x^{n}\left(p+t_{n}+q_{n}+\theta, F+g^{n}\right)\left|+M\left(p_{n}+q_{n}\right)\right| x_{t_{n}}^{n}(F+$ $\left.g^{n}\right)-\left.x_{t_{n}+p-p_{n}}^{n}\left(F+g^{n}\right)\right|_{B} \leqq 2 K L\left|p_{n}-p\right|+M\left(p_{n}+q_{n}\right)\left\{\left|x_{t_{n}}^{n}\left(F+g^{n}\right)\right|_{B}+\right.$ $\left.\left|x_{t_{n}+p-p_{n}}^{n}\left(F+g^{n}\right)\right|_{B}\right\} \leqq 2 K L\left|p_{n}-p\right|+4 H M\left(p_{n}+q_{n}\right)$, and hence we have $\left|y_{p}-w_{p}\right|_{B} \geqq\left|x_{t_{n}+r_{n}}^{n}\left(F+g^{n}\right)-u_{t_{n}+r_{n}}\right|_{B}-\left|y_{p}-y_{p}^{n}\right|_{B}-\left|y_{p}^{n}-y_{p_{n}}^{n}\right|_{B}-\mid y_{p_{n}}^{n}-$ $\left.x_{t_{n}+r_{n}}^{n}\left(F+g^{n}\right)\right|_{B}-\left|u_{t_{n}+r_{n}}-w_{p_{n}}\right|_{B}-\left|w_{p_{n}}-w_{p}\right|_{B} \geqq \delta_{1} / 2$ for all large $n$. Thus the solution $u(t)$ is totally stable.

5. Almost periodic systems. In this section, we shall assume that $B$ is separable and that $F(t, \phi)$ is almost periodic in $t$ uniformly for $\phi \in B$. Then, it is known that for any sequence $\left\{t_{n}^{\prime}\right\}$, there exists a subsequence $\left\{t_{n}\right\}$ of $\left\{t_{n}^{\prime}\right\}$ and a continuous function $G(t, \phi)$ such that $F\left(t+t_{n}, \phi\right) \rightarrow G(t, \phi)$ as $n \rightarrow \infty$ uniformly on $R \times S$, where $S$ is any compact set in $B$. We shall note that $G(t, \phi)$ also is almost periodic in $t$ uniformly for $\phi \in B$ (cf. [7]).

Corollary 5. Suppose that System (1) is regular and has the null solution which is weakly uniformly asymptotically stable. Then the null solution of (1) is uniformly asymptotically stable.

Proof. We shall show that the null solution of (1) is attracting in $H^{+}(F)$. Then the conclusion follows from Proposition 1 and Theorem, immediately.

By Proposition 1, for any $(G, R \times B) \in H^{+}(F)$ and $s \geqq 0, x_{t}\left(s, \phi^{0}, G\right)$ exists in the future and $\left|x_{t}\left(s, \phi^{0}, G\right)\right|_{B}<\delta_{0} / 2$ for all $t \geqq s$, if $\left|\phi^{0}\right|_{B}<$ $\delta\left(\delta_{0} / 4\right) / 2$, where $\delta(\cdot)$ and $\delta_{0}$ are the ones given for weak uniform asymptotic stability of the null solution of (1). Uniform stability of the null solution of (2) implies that $\left|x_{t}\left(s, \phi^{0}, G\right)\right|_{B} \rightarrow 0$ as $t \rightarrow \infty$, as required, or there exists a constant $r>0$ such that $r \leqq\left|x_{t}\left(s, \phi^{0}, G\right)\right|_{B}<\delta_{0} / 2$ for all $t \geqq s$.

Suppose that $r \leqq\left|x_{t}\left(s, \phi^{0}, G\right)\right|_{B}<\delta_{0} / 2$ for all $t \geqq s$. Then, there exists a sequence $\left\{p_{n}\right\}, p_{n} \rightarrow \infty$ as $n \rightarrow \infty$, such that $G\left(t+p_{n}, \phi\right) \rightarrow F(t, \phi)$ as $n \rightarrow \infty$ uniformly on $R \times\left\{x_{t} \mid t \geqq s\right\}^{-}$and $x\left(t+p_{n}, s, \phi^{0}, G\right) \rightarrow y(t)$ uniformly on any compact subset of $R$ as $n \rightarrow \infty$. Clearly, $y(t)$ is the solution of (1) through $\left|y_{0}\right|_{B}<\delta_{0}$ and $\left|y_{t}\right|_{B} \geqq r / 2$ for all $t \geqq 0$. However, $\left|y_{0}\right|_{B}<$ $\delta_{0}$ implies $\left|y_{t}\right|_{B} \rightarrow 0$ as $t \rightarrow \infty$, which is a contradiction. Hence the null solution of (1) is attracting in $H^{+}(F)$. 
REMARK 6. It is known that for ordinary differential equations Corollary 5 holds good without the regularity assumption. (Cf. [1], [9], [12]). The author feels that the condition "System (1) is regular" in Corollary 5 can be dropped, but is unable to prove so.

\section{REFERENCES}

[1] Z. ARtstein, Uniform asymptotic stability via the limiting equations, J. Differential Equations 27 (1978), 172-189.

[2] P. BoNDI, V. Moauro and F. Visentin, Limiting equations in the stability problem, Nonlinear Analysis. Theory, Method and Applications 1 (1977), 123-128.

[3] J. K. HALE, Dynamical systems and stability, J. Math. Anal. Appl. 26 (1969), 39-69.

[4] J. K. Hale and J. Kato, Phase space for retarded equations with infinite delay, Funkcial. Ekvac. 21 (1978), 11-41.

[5] J. Kato and T. Yoshizawa, A relationship between uniformly asymptotic stability and total stability, Funkcial. Ekvac. 12 (1969), 233-238.

[6] J. Kato, Uniformly asymptotic stability and total stability, Tôhoku Math. J. 22 (1970), 254-269.

[7] J. Kato, Stability problem in functional differential equations with infinite delay, Funkcial. Ekvac. 21 (1978), 63-80.

[8] L. Markus, Asymptotically autonomous differential systems, Contributions to Nonlinear Oscillations, Vol. 3, 17-29, Princeton Univ. Press, 1956.

[9] G. SeIfert, Almost periodic solutions and asymptotic stability, J. Math. Anal. Appl. 21 (1968), 136-149.

[10] G. R. SELL, Nonautonomous differential equations and topological dynamics, I, II, Trans. Amer. Math. Soc. 127 (1967), 241-262, 263-283.

[11] G. R. Seld, Topological Dynamics and Ordinary Differential Equations, Van Nostrand Reinhold Company, London, 1971.

[12] T. Yoshizawa, Stability Theory and the Existence of Periodic Solutions and Almost Periodic Solutions, Appl. Math. Sci., 14, Springer-Verlag, Berlin, Heidelberg and New York, 1975.

Department of Mathematics

CHIBA UNIVERSITY

CHIBA, 260

JAPAN. 
